\title{
Bandwidth Efficient Multi-Station Wireless Streaming Based on Complete Complementary Sequences
}

\author{
Chadi Khirallah, Vladimir Stanković, Lina Stanković, Yang Yang, and Zixiang Xiong
}

\begin{abstract}
Data streaming from multiple base stations to a client is recognized as a robust technique for multimedia streaming. However the resulting transmission in parallel over wireless channels poses serious challenges, especially multiple access interference, multipath fading, noise effects and synchronization. Spread spectrum techniques seem the obvious choice to mitigate these effects, but at the cost of increased bandwidth requirements. This paper proposes a solution that exploits complete complementary spectrum spreading and data compression techniques jointly to resolve the communication challenges whilst ensuring efficient use of spectrum and acceptable bit error rate. Our proposed spreading scheme reduces the required transmission bandwidth by exploiting correlation among information present at multiple base stations. Results obtained show $1.75 \mathrm{Mchip} / \mathrm{sec}$ (or $25 \%$ ) reduction in transmission rate, with only up to $6 \mathrm{~dB}$ loss in frequency-selective channel compared to a straightforward solution based solely on complete complementary spectrum spreading.
\end{abstract}

Index Terms-Data streaming, spread spectrum technology, fading, multiuser detection.

\section{INTRODUCTION}

$\mathbf{M}$ ULTI-SERVER communication, in the form of multimedia downloading and streaming in parallel from multiple sites over wireline networks, has become very attractive, so much so that many efficient solutions have been developed [1], [2], [3], [4]. This is because multi-server streaming, compared to the traditional single-server streaming, has numerous advantages, namely: (i) reconstructed video is of better quality due to path diversity gain, (ii) resilience to server failure is improved, i.e., when a server fails (for example, due to too many incoming requests), remaining servers can maintain communications, (iii) security can be enhanced by a properly designed coding scheme (e.g., two servers can send two complementary parts of the source information, with the client requiring both parts in order to recover the source).

Research on multi-server streaming has mainly focused on the fixed packet network scenario, such as streaming over the Internet. However, with the imminent large-scale deployment of the mobile WiMAX standard, which supports the possibility

Manuscript received August 28, 2007; revised December 5, 2007; accepted March 27, 2008. The associate editor coordinating the review of this letter and approving it for publication was J. Zhang.

C. Khirallah, V. Stanković, and L. Stanković are with the Dept. of Electronic and Electrical Engineering, University of Strathclyde, Glasgow, UK (e-mail: \{chadi.khirallah, vladimir.stankovic, lina.stankovic\}@eee.strath.ac.uk).

Y. Yang and Z. Xiong are with the Dept. of Electrical and Computer Engineering, Texas A\&M University, College Station, TX 77840, USA (email \{yangyang, zx $\} @$ ece.tamu.edu).

This work was supported in part by EPSRC Grant EP/E021964/2. This paper was presented in part at the Allerton-2007 Conference, Monticello, IL, Sept. 2007, and PCS-2007, Lisbon, Portugal, Nov. 2007.

Digital Object Identifier 10.1109/TWC.2009.070958 of employing two base stations (BSs) for multimedia transmission to a client device [5], wireless streaming from multiple base stations is a realistic scenario. This multi-station setup is also applicable to wireless ad hoc networks, and in wireless peer-to-peer networks, where the same or similar video content is streamed from multiple network nodes to a mobile receiver. Thus, we consider the problem of multi-station streaming over wireless channels to mobile receivers/clients. Our wireless multi-station streaming solution will enjoy smooth handover because the client is always connected to at least one BS (during transition/handover to another), and traffic load can be dynamically adjusted depending on channel conditions, e.g., when one channel is in a deep fade, the others will be loaded more heavily, thus yielding large spatial diversity gain.

Multi-station wireless streaming of data requires that base stations are located in close proximity so that the target users are within range of more than one BS at any time. This implies that spread spectrum modulation is necessary to mitigate fading, multiple access interference, and channel noise effects that arise from this setup. The main issues that must be resolved here are multiple access (since a client simultaneously receives streams from at least two BSs) and synchronization (of the incoming streams from multiple BSs). Typically, data will be spread separately at each BS; at the receiver, the streams received from different BSs are separated using despreading. Spreading provides effective interference reduction and protection, but at the expense of increased bandwidth. Indeed, better protection implies designing spreading sequences with larger redundancy or bandwidth requirements. However, often in wireless networks, the system can only afford very limited spreading. Therefore, using conventional spreading techniques will double the total sending load if two BSs are used for streaming the same content from two sites simultaneously,

In this paper, we attempt to reduce the required bandwidth by exploiting the fact that the same source, or two highly correlated sources, are streamed over two wireless channels. 'Distributed compression' is performed by puncturing the spreading sequence components, prior to transmission, depending on channel conditions. The main idea is to puncture the output stream of a BS before transmission (i.e., to remove part of spread signal) leading to reduced transmission rate. We consider two cases: (i) when the source information at the streaming BSs is identical (streaming from multiple BSs), (ii) and when the BSs are provided with correlated information (which arises, e.g., in sensor networks or stereo video applications). We also design a de-spread scheme at the decoder which combines the received streams by exploiting correlation among the sources at different BSs, and recovers the original 
data. Specifically, we propose the use of complete complementary (CC) sequences to perform spreading [6]. Our system design takes advantage of the ideal correlation properties of the CC sequences, namely, zero cross-correlation and zero outof-phase auto-correlation [7], that allow a receiver to separate mixed signals from streaming BSs and filter out delayed copies of received signals caused by multipath propagation.

$\mathrm{CC}$ sequences have already been employed in downlink and uplink wireless communication scenarios with flat- and frequency-selective fading, showing competitive performance and improved spectral efficiency compared to standard spreading sequences [8]. However, we focus on further bandwidth reduction in a multiple-station scenario by exploiting correlation information present among these BSs. The emphasis is in designing a source-independent CC coding scheme for robust delivery of highly correlated data from two or more asynchronous BSs to a mobile client. To achieve this further bandwidth reduction, straightforward application of CC sequences is not possible because of loss of orthogonality caused by puncturing. We thus develop a new encoding scheme and a corresponding iterative decoding scheme based on CC sequences that provide both multiple access and robustness to interference and fading, while removing the redundancy in the transmitted data stream. Hence our system (i) inherits the advantages of $\mathrm{CC}$ sequences over traditional spreading sequences, (ii) achieves bandwidth reduction beyond what is possible by straightforward use of $\mathrm{CC}$ sequences. The designs are independent of the source data, and can be applied to any parallel transmission such as video, audio, or still images. We show that our scheme achieves a $25 \%$ saving in bandwidth with a small performance loss compared to a standard spreading system that does not perform puncturing.

The paper is organized as follows. In the next section we briefly review key properties of CC sequences. In Section III we describe our proposed design for multi-station streaming including multi-station transmission and iterative decoder design. Our experimental results are presented in Section IV, followed by conclusions and future work in Section V.

\section{Complete Complementary Sequences}

$\mathrm{CC}$ sequences exhibit three fundamental differences compared to other well-known spreading sequences (such as Gold sequences, m-sequences, Walsh Hadamard sequences, etc.). Firstly, the mutual orthogonality of CC sequences is observed between 'sets' (of constituent sequences), rather than between constituent sequences. Secondly, the processing gain of the $\mathrm{CC}$ sequences equals to the aggregate length of all sequences in each set. Thirdly, CC sequences possess unique correlation properties with zero cross-correlation and zero out-of-phase auto-correlation for any relative shifts between two sequences. The performance of CC sequences surpasses that of any of the standard spreading sequences because of these ideal correlation properties [7], [9].

Systems based on CC sequences use the offset stacked (OS) spreading modulation technique [7] to spread data before transmitting different $\mathrm{CC}$ element sequences on orthogonal carrier frequencies. Due to the OS technique, systems are inherently capable of multirate transmission simply by shifting different number of chips between consecutive spread symbols to speed up or slow down the data rate. No complex rate matching algorithms are needed. This makes CC sequences very attractive for real-time services, e.g., video streaming, with variable rate requirements to which data rate change should be made scalable and continuous.

According to [7], in order for $N$ sets, each made up of $N$ sequences, to be $\mathrm{CC}$ sequences of order $N$, they must satisfy the following properties: (i) the sum of the $N$ auto-correlation functions for each set is zero for any shift except the zero shift (this set is called 'auto-complementary sequence of order $N$ '); (ii) the sum of the $N$ cross-correlation functions between two sets of $N$ sequences is zero for any shift (each of these two sets is called 'cross-complementary sequence of order $N^{\prime}$ '). $\mathrm{CC}$ sequences can be generated as in [9].

Traditionally, each user in a cellular communication system is assigned a unique set of CC sequences. Since the total number of available sets is $N=\sqrt{k}=2^{j}, j=1,2, \ldots$, with $k$ being the length of each element sequence, then the total number of users in one cell of the system is $N$. For example, when $k=4$, the total number of available CC sets is $N=2$; the first set, $X$, assigned to the first user, consists of two element sequences $\mathbf{w}_{11}=[+,+,+,-]$ and $\mathbf{w}_{12}=[+,-,+,+]$, while the second set, $Y$, assigned to the second user, contains $\mathbf{w}_{21}=[+,+,-,+]$ and $\mathbf{w}_{22}=$ $[+,-,-,-]$ (here, "+" denotes +1 and "-" is -1 ). The autocorrelation functions for $\mathrm{CC}$ sequence sets $X$ and $Y$ are $\Psi_{X X}=\mathbf{w}_{11} \oplus \mathbf{w}_{11}+\mathbf{w}_{12} \oplus \mathbf{w}_{12}=[0,0,0,8,0,0,0]$ and $\Psi_{Y Y}=\mathbf{w}_{21} \oplus \mathbf{w}_{21}+\mathbf{w}_{22} \oplus \mathbf{w}_{22}=[0,0,0,8,0,0,0]$, where $\oplus$ denotes the shift-and-add operation. The cross-correlation functions between $\mathrm{CC}$ sets $X$ and $Y$ are the sum of all cross-correlation functions between any pair of constituent sequences in those sets $\Psi_{X Y}=\mathbf{w}_{11} \oplus \mathbf{w}_{21}+\mathbf{w}_{12} \oplus \mathbf{w}_{22}=$ $[0,0,0,0,0,0,0]$.

$\mathrm{CC}$ element sequences can be sent to the receiver either in parallel using different orthogonal carriers, such as in multicarrier transmission, or serially where all constituent element sequences are sent using the same carrier. In this paper, the former approach is adopted with two carrier frequencies.

\section{PROposed SySTEM}

This section describes our system set-up for efficiently streaming data from multiple BSs over wireless links. Only $N=2$ BSs are used as example in this paper for ease of presentation, but the methodology and results are more general and can be extended to $N>2$ BSs. The maximum number of streaming BSs is bounded to the maximum number of available CC sets $N$ or vice versa. We address the scenario where the BSs stream identical or correlated source data to the client. It is assumed that the client is in the range of these BSs during the entire communication period, and the influence of all other clients is treated as noise.

\section{A. Spreading at the base station}

Spreading by CC sequences, at each of the BSs, is performed after data compression and possibly channel coding, and ensures the transmitted signal streams are robust to the 


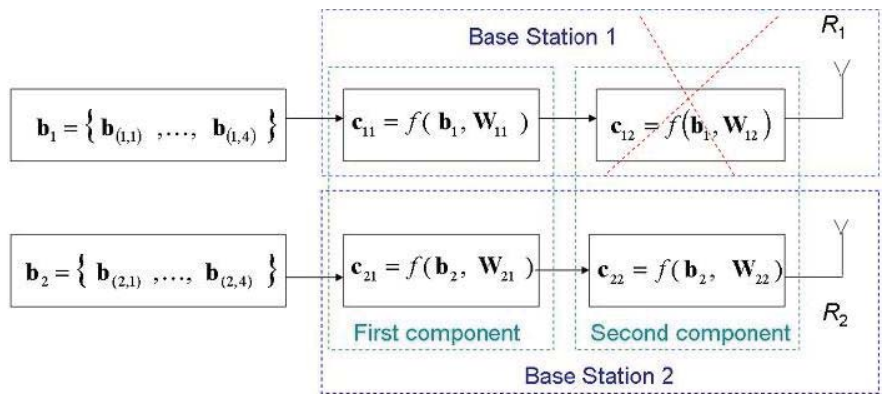

Fig. 1. An example of spreading two source blocks $\mathbf{b}_{1}$ and $\mathbf{b}_{2}$, which consist of four symbols each, using four CC sequences of length $k=4$. The second component of the spread signal transmitted from BS1, $\mathbf{c}_{12}$, is punctured. The two BSs transmit at rates $R_{1}$ and $R_{2}$, respectively.

time shifts induced either by a time-delay spread of a multipath fading channel and/or asynchronous transmission from multiple BSs to a client. Source sequences are partitioned into source blocks $\mathbf{b}_{1}$ and $\mathbf{b}_{2}$ of length $k$ symbols (e.g., bits) present at BS1 and BS2, respectively. As mentioned in Section II, in conventional CC spreading, the transmitted spread signal consists of $N=\sqrt{k}$ components that are transmitted in parallel over $N$ orthogonal carrier frequencies. In this twostation example, we use a CC element sequence of length four, i.e., $k=4$ and $N=2$. Applying the spread-and-shift of the OS spreading technique, for each block of four data symbols, seven chips are transmitted at each of the two frequencies from each BS or 14 chips in total over both carriers. BS1 uses CC sequences $\mathbf{w}_{11}$ and $\mathbf{w}_{12}$, whilst $\mathrm{BS} 2$ uses sequences $\mathbf{w}_{21}$ and $\mathbf{w}_{22}$ for spreading. BS1 encodes $\mathbf{b}_{1}$ with $\mathbf{w}_{11}$ into the spread sequence $\mathbf{c}_{11}$, as shown in Fig. 1, and sends it on frequency $f_{1}$; at the same time, it uses $\mathbf{w}_{12}$ to spread the same source block into $\mathbf{c}_{12}$, and sends it on frequency $f_{2}$. Similarly, BS2 encodes $\mathbf{b}_{2}$ using $\mathbf{w}_{21}$ and $\mathbf{w}_{22}$ into $\mathbf{c}_{21}$ and $\mathbf{c}_{22}$, respectively, and transmits them on frequency $f_{1}$ and $f_{2}$, respectively.

Let $G$ be the spreading factor defined as the ratio between the length (number of chips) of the output sequence $\mathbf{c}_{i j}, i, j=$ $\{1,2\}$, and the length of the source sequence $k$ per carrier frequency. The transmission rate $R_{i}$ is defined as the total number of chips sent per source symbol from BS $i(i=1,2)$ on both carrier frequencies. For the conventional spreading case [7], each user is assigned one set of $N=2$ element sequences to be used to spread all source symbols. This results in transmission rate $R_{i}=N G$. For the above example we have $k=4, G=7 / 4$ and $R_{i}=14 / 4=3.5$ for each of the two carrier frequencies; the total rate from the two BSs to the client is then $R=R_{1}+R_{2}=7$ chips per source symbol.

However, the number of transmitted chips can be reduced by puncturing some of the transmitted chips (see Fig. 1), thus facilitating rate/bandwidth adaptability. The number of punctured chips and the best puncturing pattern depend on the quality of the transmission channel between the BSs and the client. An example of the rate reduction process is shown in Fig. 1, where BS1 sends only the first spread signal component $\mathbf{c}_{11}$ and BS2 sends both $\mathbf{c}_{21}$ and $\mathbf{c}_{22}$. This results in a total transmission rate of $R=R_{1}+R_{2}=3 / 2 N G$, which in the case of $k=4, N=2$, and $G=7 / 4$ results in $R=21 / 4=5.25$ chips per source symbol. In order to evenly load both BSs, for the next source block, BS1 transmits both its component sequences, $\mathbf{c}_{11}$ and $\mathbf{c}_{12}$, while BS2 sends only one, i.e., $\mathbf{c}_{21}$.

In general, different rate allocations between the two BSs are possible, as long as the total transmission rate $R$ is large enough to decode; this depends on the available channels between the BSs and the client and the correlation among the sources. The client can optimize rate allocation among the BSs by exploiting channel information obtained via feedback. The client can first sort the channels (from each BS) according to their highest estimated channel coefficient values, and then report this result to the BSs, which perform puncturing accordingly. Hence, the best puncturing pattern will depend on channel statistics collected at the client side. We do not focus in this paper on finding the optimal puncturing pattern based on channel statistics, but we use the intuitive fact that BSs with better channels should puncture more signals in order to maximize performance.

Although the idea of puncturing may seem straightforward, simply puncturing spread chips of one or more transmitted signal components will result in loss of mutual orthogonality between the sets of $\mathrm{CC}$ sequences. Note that orthogonality is established between sets of all sequences used in spreading [7]. Hence, we cannot use conventional despreading at the client. We propose a novel despreading decoder, which is iterative, that enables recovery of mutual orthogonality. Such a decoder, described below, needs to exploit correlation among the two source sequences and remove redundancy in the transmitted signals.

\section{B. Client-side detection: Iterative decoder design}

At the client side, estimation of the original streams is performed iteratively. The gist of our iterative decoder is best explained by means of an example.

Let $\hat{h}_{1}$ and $\hat{h}_{2}$ denote the estimated channel coefficients from BS1 and BS2 to the client, respectively. In this example, we assume flat fading for simplicity, and perfect channel knowledge at the client. When we implement the decoder for frequency-selective fading channel, we use an additional Successive Interference Canceller (SIC) to eliminate Intersymbol interference (ISI) resulting at each despread stage [9]. Suppose that the client reported channel coefficients $\hat{h}_{1}$ and $\hat{h}_{2}$ to the BSs and that $\left\|\hat{h}_{1}\right\|^{2}>\left\|\hat{h}_{2}\right\|^{2}$. Since the channel from BS1 is better, BS1 decides to send only one spread sequence, say $\mathbf{c}_{11}$, while BS2 sends both $\mathbf{c}_{21}$ and $\mathbf{c}_{22}$. Let the signals received at the decoder at frequency $f_{1}$ and $f_{2}$ be: $\mathbf{r}_{1}=h_{1} \mathbf{c}_{11}+h_{2} \mathbf{c}_{21}+\mathbf{z}_{1}$ and $\mathbf{r}_{2}=0+h_{2} \mathbf{c}_{22}+\mathbf{z}_{2}$, respectively, where $\mathbf{z}_{1}$ and $\mathbf{z}_{2}$ are white Gaussian noise, each of unit power and independent of sent signals and each other.

The decoder in Fig. 2 starts by despreading received signals $\mathbf{r}=\left\{\mathbf{r}_{1}, \mathbf{r}_{2}\right\}$ using conventional CC sequences correlators [9]. The first signal component is transmitted from BS1 on carrier frequency $f_{1}$ and is contained only in the received signal $\mathbf{r}_{1}$. Hence, the decoder obtains the estimate of $\mathbf{b}_{1}$ sequence from $\mathbf{r}_{1}$ at the first iteration as $\hat{\mathbf{b}}_{1}(1)$. Let $l$ be initialized to 1 . Then, at the $l$-th iteration, the decoding algorithm is as follows:

- The estimated sequence $\hat{\mathbf{b}}_{1}(l)$ is spread using BS1's sequence $\mathbf{w}_{11}$ giving an estimate of $\mathbf{c}_{11}$, as $\hat{\mathbf{c}}_{11}(l)$, and the result is multiplied by $\hat{h}_{1}$. This is then canceled out from the received signal $\mathbf{r}_{1}$, that is, $\hat{\mathbf{r}}_{1}=\mathbf{r}_{1}-\hat{h}_{1} \hat{\mathbf{c}}_{11}(l)$. 


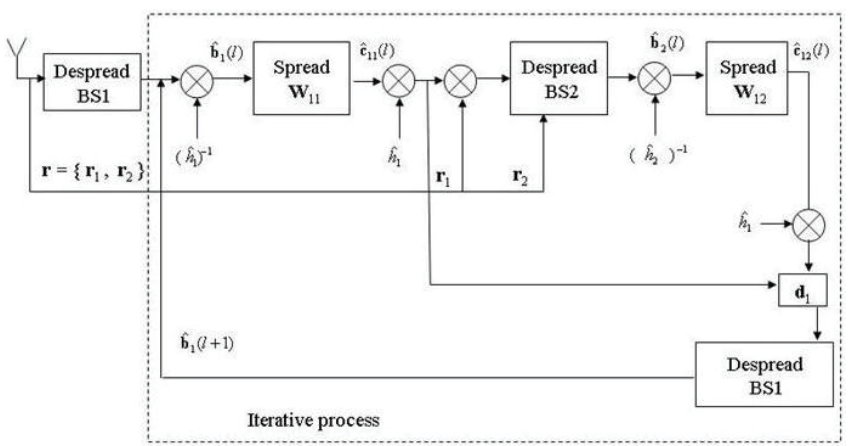

Fig. 2. Block diagram of the proposed iterative decoder.

Next, BS2's source block sequence is estimated as $\hat{\mathbf{b}}_{2}(l)$ by conventional despreading from $\hat{\mathbf{r}}_{1}$.

- $\hat{\mathbf{b}}_{2}(l)$ instead of $\hat{\mathbf{b}}_{1}(l)$ (since the sources are assumed to be highly correlated) is further spread using the second $\mathrm{CC}$ sequence assigned to $\mathrm{BS} 1, \mathbf{w}_{12}$, to estimate the second signal component $\mathbf{c}_{12}$ (which was punctured at $\mathrm{BS} 1$ ); this way, $\hat{\mathbf{c}}_{12}(l)$ is obtained, which is then multiplied by the channel coefficient $\hat{h}_{1}$. (Note that, in the case when the decoder knows the correlation between the source sequences $\mathbf{b}_{1}$ and $\mathbf{b}_{2}$, prior to spreading, it can pass $\hat{\mathbf{b}}_{2}(l)$ through a "virtual" correlation channel to obtain $\hat{\mathbf{b}}_{1}(l)$; however, in this paper we assume no such knowledge at the decoder.)

- The symbols belonging to BS1 are extracted, as $\hat{\mathbf{b}}_{1}(l+$ 1 ), by despreading the constructed sequence $\mathbf{d}_{1}=$ $\left\{\hat{h}_{1} \hat{\mathbf{c}}_{11}, \hat{h}_{1} \hat{\mathbf{c}}_{12}(l+1)\right\}$.

- Counter $l$ is incremented, and the above steps are repeated until a satisfactory bit error rate (BER) performance for estimated $\mathbf{b}_{1}$ and $\mathbf{b}_{2}$ is reached.

The two source sequences at the two BSs can be identical, or they can be two correlated sequences, e.g., two different views of the same scene in stereo video coding. In the next section, we provide simulation results for both cases.

\section{Simulation Results}

In all our simulations we assume uniform random binary sources at the two streaming BSs, whose correlation is modeled as a binary symmetric channel (BSC) with crossover probability $p ; p=0$ implies that both sources are identical. The source rate is set to $1 \mathrm{Msymbol} / \mathrm{sec}$. We use CC element sequences of length $k=4$ and two carrier frequencies, such that $G=7 / 4$. All our results are averaged over 1,000,000 symbols transmitted per BS. BSs stream data asynchronously - simulated by an inter-BS shift of one chip - to a mobile client with relative client-BS speeds of 30 and $120 \mathrm{~km} / \mathrm{hr}$. The combined transmission rate at the client for the proposed system, $R=R_{1}+R_{2}=5.25 \mathrm{Mchip} / \mathrm{sec}$, is obtained by alternatingly puncturing output signal components, $c_{i j}$, of the two BSs, as described in Section II. Results for a conventional $\mathrm{CC}$ spreading system without puncturing (hence, the combined rate at the client is $R=7 \mathrm{Mchip} / \mathrm{sec}$, where $R_{1}=R_{2}=3.5$ Mchip/sec) are included as a benchmark.

Our channel model is set up with the following parameters: (i) a frequency-selective Rayleigh fading distribution; (ii) each

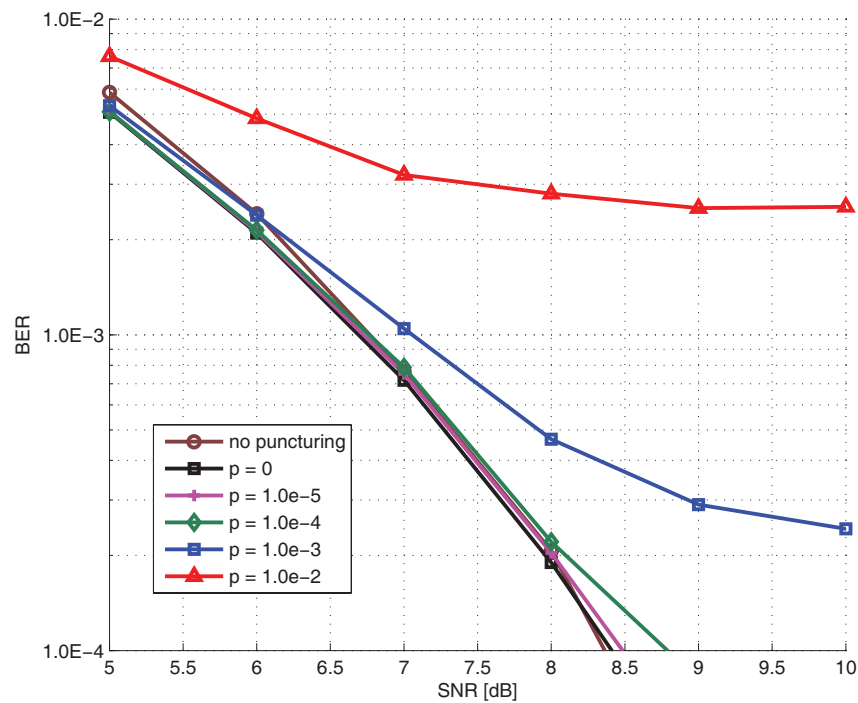

Fig. 3. BER vs SNR for the AWGN channel.

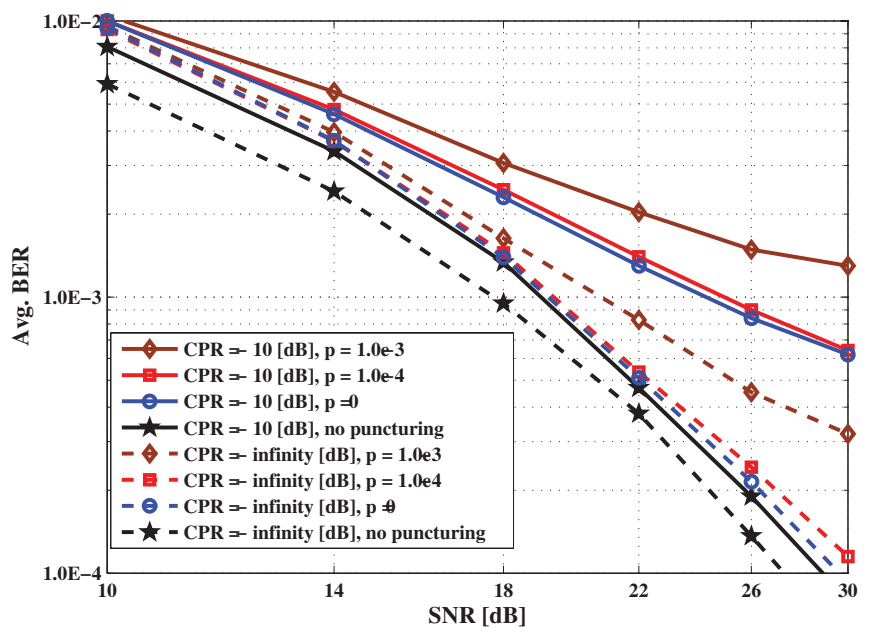

Fig. 4. BER vs SNR for relative client-BS speed of $30 \mathrm{~km} / \mathrm{hr}$.

sub-channel between a BS and a client has two paths with the second path delayed by one chip. Perfect channel estimation is assumed. The channel power ratio (CPR) is defined as the average power ratio between the second and first paths.

We show the results for: (i) conventional CC system (no compression) with two identical sources $(p=0)$, (ii) compression of identical sources $(p=0)$, and (iii) compression of correlated sources $\left(p=10^{-4}\right.$ and $\left.p=10^{-3}\right)$.

Fig. 3 shows BER results for the Additive White Gaussian Noise (AWGN) case. The channels from the two BSs are simulated with the same signal-to-noise ratio (SNR). As observed, there is almost no performance loss when puncturing is used. Note that good results are obtained as long as the crossover probability $p$ is less than or equal to 0.001 . It is obvious that the puncturing rate used is too high for $p=0.01$, since the curves hit the error floor at BER $>10^{-3}$.

For the fading case we allow for the fact that the channels between the two BSs and client may have different SNR. This is simulated in our experiments as SNR and SNR-1dB from BS1 and BS2, respectively. BER averaged over two streams as a function of SNR with CPR $=-10 \mathrm{~dB}$ (frequency-selective) 


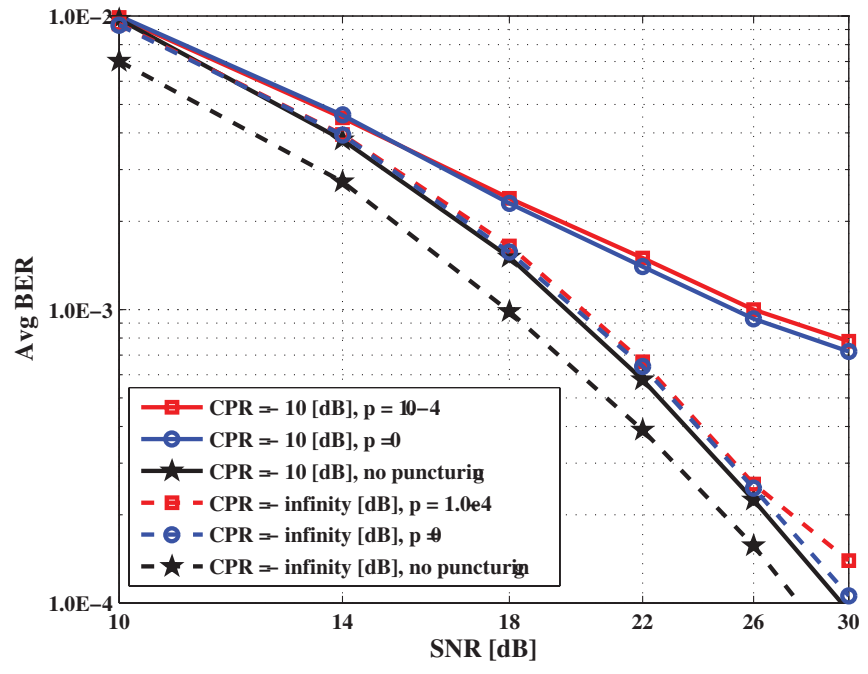

Fig. 5. BER vs SNR for relative client-BS speed of $120 \mathrm{~km} / \mathrm{hr}$.

and $\mathrm{CPR}=-\infty \mathrm{dB}$ (flat-fading channel) with relative clientBS speeds of 30 and $120 \mathrm{~km} / \mathrm{hr}$ are shown in Figs. 4 and 5, respectively. We observe in Fig. 4 that the proposed system achieves almost error-free performance with $\mathrm{BER} \leq 10^{-3}$, $\mathrm{CPR}=-10 \mathrm{~dB}$ and $p \leq 10^{-4}$ at $\mathrm{SNR} \approx 25 \mathrm{~dB}$, with degradation of almost $6 \mathrm{~dB}$ from the flat fading case. Similar results are obtained for the $120 \mathrm{~km} / \mathrm{hr}$ speed case, as shown in Fig. 5. This demonstrates the robust system performance with variable client-BS speeds. The loss with respect to the conventional system without compression is larger for the frequency-selective case $(\mathrm{CPR}=-10 \mathrm{~dB})$. This degradation is due to the error propagation problem associated with the single iteration feedback stage used in the SIC algorithm.

\section{CONClusions AND Future Work}

In this paper, a solution for data streaming from multiple base stations is proposed that is robust to multiple access interference, fading and asynchronous parallel transmissions over frequency-selective wireless channels. The properties of Complete Complementary spreading sequences are exploited together with correlation information among sources. A new low complexity iterative decoder is developed that combines the streams received from both wireless BSs to recover from the loss of mutual orthogonality caused by puncturing spread sequences at the BS. Our design achieves $1.75 \mathrm{Mchip} / \mathrm{sec}$ (or $25 \%$ ) rate reduction at the client side, over a conventional spreading system (without puncturing) with acceptable loss in BER performance.

In future work we intend to look further into the challenges of multimedia streaming over multiple BSs, including distributed compression, optimal rate and power allocation among the BSs, and the effects of correlated fading channels, which all need to be resolved before practical deployment of the system. Short term investigations include the possibility of: (i) achieving a more flexible rate trade-off among BSs using longer $\mathrm{CC}$ sequences and variable puncturing rates (in this paper a puncturing rate of $50 \%$ is used), (ii) improving BER performance using more iterations at the decoder, channel coding, and a SIC more robust to error propagation.

\section{REFERENCES}

[1] J. G. Apostolopoulos, T. Wong, W. Tan, and S. Wee, "On multiple description streaming with content delivery networks," in Proc. IEEE INFOCOM-2002, New York, NY, June 2002.

[2] J. P. Wagner and P. Frossard, "Adaptive and robust media streaming over multiple channels with burst losses," in Proc. EUSIPCO-2007 15th European Signal Processing Conference, Poznan, Poland, Sept. 2007.

[3] P. Chou, V. Padmanabhan, H. Wang, and K. Sripanidkulchai, "Distributing streaming media content using cooperative networking," in Proc. ACM NOSSDAV, Miami Beach, FL, May 2002.

[4] T. Nguyen and A. Zakhor, "Multiple sender distributed video streaming," IEEE Trans. Multimedia, vol. 6, pp. 315-326, Apr. 2004.

[5] J. Wang, M. Venkatachalam, and Y. Fang, "System architecture and cross-layer optimization of video broadcast over WiMAX," IEEE J. Select. Areas Commun., vol. 25, pp. 712-721, May 2007.

[6] N. Suehiro and M. Hatori, "N-shift cross-orthogonal sequences," IEEE Trans. Inform. Theory, vol. IT-34, pp. 143-146, 1988.

[7] H. H. Chen, J. F. Yeh, and N. Suehiro, "A multicarrier CDMA architecture based on orthogonal complementary codes for new generations of wideband wireless communications," IEEE Commun. Mag., vol. 39 no. 10 , pp. 126-135, Oct. 2001.

[8] E. H. Dinan and B. Jabbari, "Spreading codes for direct sequence CDMA and wideband CDMA cellular networks," IEEE Commun. Mag., pp. 48-54, Sept. 1999.

[9] C. Khirallah, P. Coulton, H. Rashvand, and N. Zein, "Multi-user MIMO CDMA systems using complete complementary sequences," IEE Proc. Commun., vol. 153, no. 4, pp. 533-540, Aug. 2006. 